\title{
Moving Unstable Particles and Special Relativity
}

\author{
Eugene V. Stefanovich \\ 1763 Braddock Court, San Jose, CA 95125, USA \\ Correspondence should be addressed to Eugene V. Stefanovich; eugenev@synopsys.com \\ Received 5 December 2017; Accepted 12 March 2018; Published 19 April 2018 \\ Academic Editor: Krzysztof Urbanowski
}

Copyright (C) 2018 Eugene V. Stefanovich. This is an open access article distributed under the Creative Commons Attribution License, which permits unrestricted use, distribution, and reproduction in any medium, provided the original work is properly cited. The publication of this article was funded by SCOAP ${ }^{3}$.

\begin{abstract}
In Poincaré-Wigner-Dirac theory of relativistic interactions, boosts are dynamical. This means that, just like time translations, boost transformations have a nontrivial effect on internal variables of interacting systems. In this respect, boosts are different from space translations and rotations, whose actions are always universal, trivial, and interaction-independent. Applying this theory to unstable particles viewed from a moving reference frame, we prove that the decay probability cannot be invariant with respect to boosts. Different moving observers may see different internal compositions of the same unstable particle. Unfortunately, this effect is too small to be noticeable in modern experiments.
\end{abstract}

\section{Introduction}

Time dilation is one of the most spectacular predictions of special relativity. This theory predicts that any timedependent process slows down by the universal factor of $1 / \sqrt{1-v^{2} / c^{2}} \equiv \cosh \theta$ when viewed from a reference frame moving with the speed $v$ (and rapidity $\theta$ ). The textbook example of such a time-dependent process is the decay law $\Upsilon(0, t)$ of an unstable particle at rest. The function $\Upsilon(0, t)$ is the probability of finding the unstable particle at time $t$, if it was prepared with $100 \%$ certainty at time $t=0$. Then, according to special relativity, the decay law of a moving particle should be exactly $\cosh \theta$ times slower:

$$
\Upsilon^{S R}(\theta, t)=\Upsilon\left(0, \frac{t}{\cosh \theta}\right) .
$$

Indeed, this prediction was confirmed in numerous measurements [1-4]. The best accuracy of $0.1 \%$ was achieved in experiments with relativistic muons $[5,6]$.

However, the exact validity of (1) is still a subject of controversy. One point of view [7-9] is that special-relativistic time dilation was derived in the framework of classical theory and may not be directly applicable to unstable particles, which are fundamentally quantum systems without well-defined masses, velocities, positions, and so on.
However, such a quantum clock as an unstable particle cannot be at rest (i.e., cannot have zero velocity or zero momentum) and simultaneously be at a definite point (due to the quantum uncertainty relation). So, the standard derivation of the moving clock dilation is inapplicable for the quantum clock. The related quantum-mechanical derivation must contain some reservations and corrections. Shirokov [10]

Indeed, detailed quantum-mechanical calculations [8, $10-12$ ] suggest that (1) is not accurate, and that corrections to this formula should be expected, especially at large times, exceeding multiple lifetimes. Although these corrections are too small to be observed in modern experiments, their presence casts doubt on the limits of applicability of Einstein's special relativity.

Unfortunately, the corrections to (1) were derived in [8, 10-12] under certain assumptions and approximations. So, the question remains whether one can design a relativistic model in which the decay slowdown will acquire exactly the form (1) demanded by special relativity $[13,14]$ ?

In order to answer this question we will analyze the status of interactions in special relativity from a more general point of view. We are going to prove that under no circumstances the decay law transforms with respect to boosts exactly as in (1). 
TABLE 1: Inertial transformations.

\begin{tabular}{lcccc}
\hline Transformation & Type & Parameter & Generator & Meaning of generator \\
\hline Space translation & Kinematical & Distance $\mathbf{a}$ & $\mathbf{P}=\mathbf{P}_{0}$ & Total momentum \\
Rotation & Kinematical & Angle $\boldsymbol{\varphi}$ & $\mathbf{J}=\mathbf{J}_{0}$ & Total angular momentum \\
Time translation & Dynamical & Time $t$ & $H=H_{0}+V$ & Total energy (Hamiltonian) \\
Boost & Dynamical & Rapidity $\boldsymbol{K}$ & $\mathbf{K}=\mathbf{K}_{0}+\mathbf{Z}$ & Boost operator \\
\hline
\end{tabular}

\section{Materials and Methods}

2.1. Inertial Transformations. The theory of relativity tries to connect views of different inertial observers. The principle of relativity says that all such observers are equivalent; that is, two inertial observers performing the same experiment will obtain the same results.

There are four classes of inertial transformations, space translations, time translations, rotations, and boosts, and their actions on observed systems differ very much (see Table 1). For example, it is easy to describe the results of space translations and rotations. An observer displaced by the 3-vector a sees all atoms in the Universe simply shifted in the opposite direction $-\mathbf{a}$. This shift is absolutely exact and universal. It applies to all systems, however complicated. The same can be said about rotations. One can switch to the point of view of the rotated observer by simply rotating all atoms in the Universe. For example, rotation through the angle $\varphi$ about the $z$-axis results in the transformation of coordinates

$$
\begin{aligned}
& x^{\prime}=x \cos \varphi-y \sin \varphi \\
& y^{\prime}=y \cos \varphi+x \sin \varphi \\
& z^{\prime}=z
\end{aligned}
$$

which is independent on the composition of the observed system and on its interactions. Due to this exact universality, we can regard space translations and rotations as purely geometrical or kinematical transformations.

Time translation is also an inertial transformation, because repeating the same experiment at different times will not change the outcome. However, this transformation is by no means kinematical. Time evolutions of interacting systems can be very complicated. Their description requires intimate knowledge of the system's composition, state, and interactions acting between system's parts. We will say that time translations are dynamical inertial transformations.

Now, what about boosts? Are they kinematical or dynamical? In nonrelativistic classical physics boosts are definitely regarded as kinematical, they simply change velocities of all atoms in the Universe. However, things become more complicated in relativistic physics, as we shall see below.

2.2. Boosts in Special Relativity. Description of boost transformations is the central subject of special relativity. Einstein based his approach on the already mentioned relativity postulate and on his second postulate about the invariance of the speed of light. It is remarkable how all results of special relativity can be derived from these two simple and undeniable statements.
Consider the light clock shown in Figure 1(a). It consists of two parallel mirrors and the light pulse reflecting back and forth between them. The period of the clock at rest is equal to $\tau=2 t=2 l / c$. If the clock is moving, as in Figure $1(\mathrm{~b})$, the distance traveled by the light pulse increases to $l^{\prime}=2 c t^{\prime}=$ $2 \sqrt{l^{2}+\left(v t^{\prime}\right)^{2}}$. Solving this system of equations with respect to the clock period, we obtain

$$
\tau^{\prime}=2 t^{\prime}=\frac{\tau}{\sqrt{1-v^{2} / c^{2}}}=\tau \cosh \theta .
$$

So, the moving clock runs $\cosh \theta$ times slower than the clock at rest. This is the time dilation effect that we used in (1).

Let us now consider the same clock oriented parallel to its velocity, as in Figure 2. The clock's rate should not depend on its orientation, so we already know the period of this clock in motion (3). Taking into account the invariance of the speed of light, this result can be achieved only if the distance between the two mirrors decreases. The corresponding system of equations is

$$
\tau^{\prime}=t_{1}+t_{2}=\frac{\left(l^{\prime}+v t_{1}\right)}{c}+\frac{\left(l^{\prime}-v t_{2}\right)}{c} .
$$

Solving with respect to $l^{\prime}$, we obtain the familiar length contraction formula:

$$
l^{\prime}=l \sqrt{1-\frac{v^{2}}{c^{2}}}=\frac{l}{\cosh \theta} .
$$

Formulas (3) and (5) already imply that no material object can move faster than the speed of light. Otherwise, the factor $\sqrt{1-v^{2} / c^{2}}$ would become imaginary, which is absurd.

We can also make a clock, in which, instead of the light pulse, we have a massive steel ball bouncing between the two mirrors. The ball's speed $w$ is less than $c$, and the speed invariance postulate does not apply to $w$. Nevertheless, we expect this clock to obey the same time dilation and length contraction rules as derived above. Then, for consistency, we have to modify the classical velocity transformation law. For example, if the resting clock in Figure 2(a) had ball's velocities $\pm w$, then the moving clock in Figure 2(b) should have velocities

$$
\begin{aligned}
& w_{1}=\frac{w+v}{1+w v / c^{2}} \\
& w_{2}=\frac{-w+v}{1-w v / c^{2}} .
\end{aligned}
$$




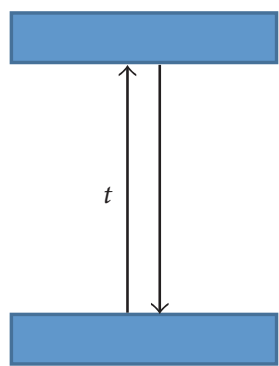

(a)

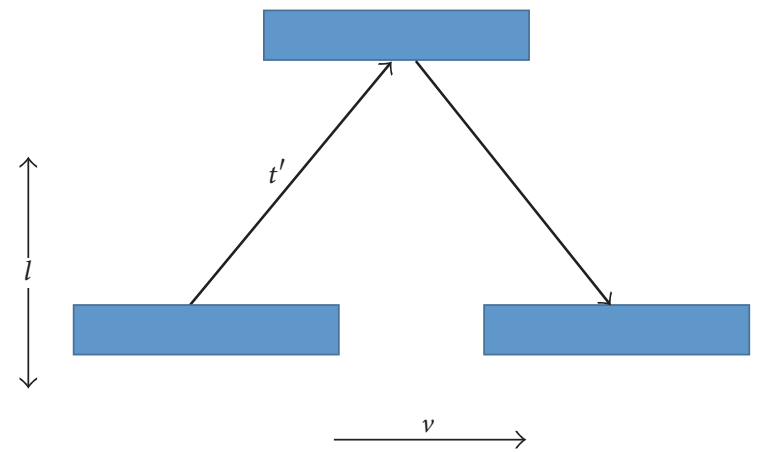

(b)

FIGURE 1: Light clock: (a) at rest and (b) in motion perpendicular to the clock's axis.

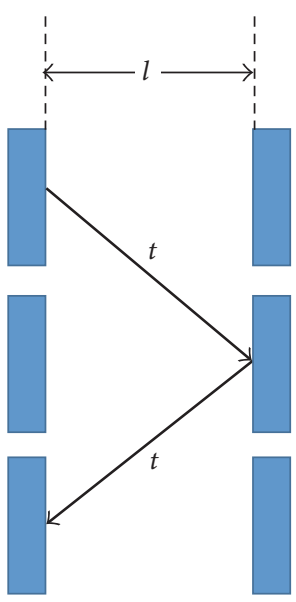

(a)

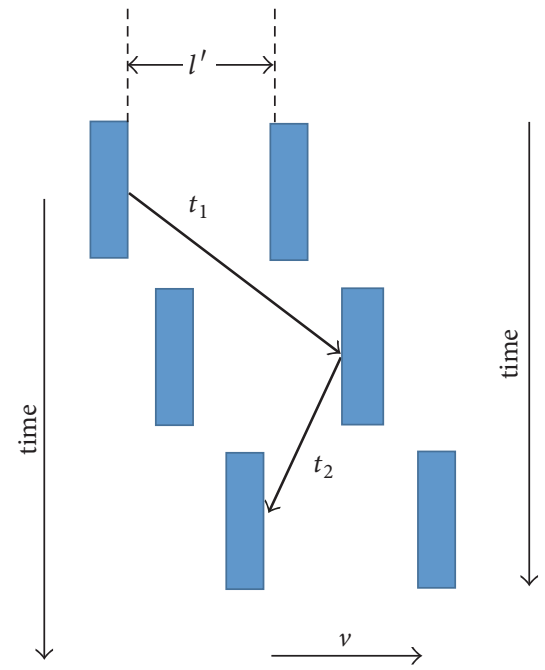

(b)

Figure 2: Light clock: (a) at rest and (b) in motion parallel to the clock's axis. The time evolution is shown in three frames stacked up vertically.

Indeed, it is not difficult to verify that these values solve the system of equations

$$
\begin{aligned}
w_{1} t_{1} & =l^{\prime}+v t_{1} \\
-w_{2} t_{2} & =l^{\prime}-v t_{2} \\
t_{1}+t_{2} & =\left(\frac{2 l}{w}\right) \cosh \theta
\end{aligned}
$$

that describe the movement of the ball during one clock period.

As a consistency check, by applying the velocity addition law (6) to the photon moving with the speed of light $(w=c)$, we return back to Einstein's light speed invariance postulate

$$
w_{1}=\frac{c+v}{1+v / c}=c
$$

Special relativity explains how all these particular results can be generalized into Lorentz transformations for the times and positions of events. For example, any event having 4-coordinates $(t, x, y, z)$ in the rest frame will have other 4coordinates

$$
\begin{aligned}
& t^{\prime}=t \cosh \theta-\left(\frac{x}{t}\right) \sinh \theta \\
& x^{\prime}=x \cosh \theta-c t \sinh \theta \\
& y^{\prime}=y \\
& z^{\prime}=z
\end{aligned}
$$

in the frame moving with velocity $v=c \tanh \theta$ along the $x$ axis. The linear character and the exact universality of these formulas are similar to transformations of 3-coordinates under rotations (2). So, it is tempting to continue this analogy and to introduce the idea of the 4D Minkowski space-time, whose points constitute physical events, and where boosts are represented by purely geometrical (kinematical) pseudorotations.

However, it is important to note that all the above derivations used model systems without interactions. The second Einstein postulate is formally applicable only to light pulses and events associated with them. So, strictly speaking, we are not allowed to extend results of special relativity 
beyond corpuscular optics. In addition, one can show [15] that Lorentz transformations (10) can be extended to special events, such as intersections of particle trajectories, involving massive noninteracting particles, for example, our steel ball and mirrors. (Of course, reflections of light pulses or steel balls from the mirrors do involve interactions, but in our idealized thought experiments we can assume that these processes take negligibly short times.)

How can we be confident that the same conclusions apply to interacting systems? For example, what if the steel ball is bouncing between plates of a charged capacitor? Can we be sure that Lorentz formulas (10) still apply?

Here we meet the following fork in the road. On the one hand, we can choose to postulate that the laws of special relativity established above are valid independent of interactions. Then boosts should be rigorously kinematical, just as space translations and rotations. This nonobvious postulate is tacitly assumed in all textbooks. In particular, it was used in numerous attempts [16-22] to derive Lorentz transformations from the first Einstein postulate only.

Alternatively, we can assume that, similar to time translations, boosts are dynamical; that is, they involve interactions, and their actions cannot be expressed by simple universal formulas, like (10). We will discuss these two possibilities in Section 3. However, before doing that, in the rest of this section, we are going to recall the fundamentals of relativistic quantum theory pioneered by Wigner and Dirac. This theory is based on the extremely important fact that inertial transformations form the Poincaré group.

2.3. Representations of the Poincaré Group in Quantum Mechanics. We are interested in application of inertial transformations to quantum systems. Properties of such systems are described by objects in the Hilbert space $\mathscr{H}$, such as state vectors and Hermitian operators of observables. So, we have to define the action (a.k.a. representation) of inertial transformations in $\mathscr{H}$. Operators of this representation $U_{g}$ must preserve quantum-mechanical probabilities, so these operators have to be unitary [23]. This brings us to the classical mathematical problem of constructing unitary representations of the Poincaré group in the given Hilbert space $\mathscr{H}$ [24].

Important role is played by the so-called "infinitesimal transformations" or generators. They are represented by Hermitian operators in $\mathscr{H}$ (see Table 1). Unitary representatives $U_{g}$ of finite transformations can be expressed by exponential functions of the Hermitian generators. For example, a general inertial transformation $g$ is a composition of (boost $\boldsymbol{\theta}) \times$ $($ rotation $\varphi) \times($ space translation $\mathbf{a}) \times($ time translation $t)$. It is represented by the following product of unitary exponents:

$$
U_{g}=e^{-(i c / \hbar) \mathbf{K} \cdot \boldsymbol{\theta}} e^{-(i / \hbar) \mathbf{J} \cdot \boldsymbol{\varphi}} e^{-(i / \hbar) \mathbf{P} \cdot \mathbf{a}} e^{(i / \hbar) H t} .
$$

Commutators of the Hermitian generators are fully determined by the structure of the Poincaré group $[25,26]$

$$
\begin{aligned}
& {\left[J_{i}, P_{j}\right]=i \hbar \sum_{k=1}^{3} \epsilon_{i j k} P_{k}} \\
& {\left[J_{i}, J_{j}\right]=i \hbar \sum_{k=1}^{3} \epsilon_{i j k} J_{k}}
\end{aligned}
$$

$$
\begin{aligned}
& {\left[J_{i}, K_{j}\right]=i \hbar \sum_{k=1}^{3} \epsilon_{i j k} K_{k}} \\
& {\left[P_{i}, P_{j}\right]=\left[J_{i}, H\right]=\left[P_{i}, H\right]=0} \\
& {\left[K_{i}, K_{j}\right]=-\frac{i \hbar}{c^{2}} \sum_{k=1}^{3} \epsilon_{i j k} J_{k}} \\
& {\left[K_{i}, P_{j}\right]=-\frac{i \hbar}{c^{2}} H \delta_{i j}} \\
& {\left[K_{i}, H\right]=-i \hbar P_{i} .}
\end{aligned}
$$

2.4. Hilbert Space of Unstable Particle. According to Wigner and Weinberg $[24,26]$, the Hilbert space $\mathscr{H}^{(i)}$ of each stable elementary particle carries an unitary irreducible representation $U_{g}^{(i)}$ of the Poincaré group. The Hilbert space of an $\mathrm{N}$-particle system is constructed as a tensor product (with proper (anti)symmetrization) of one-particle spaces

$$
\mathscr{H}^{N}=\mathscr{H}^{(1)} \otimes \mathscr{H}^{(2)} \otimes \cdots \otimes \mathscr{H}^{(N)} .
$$

In the formalism with varied numbers of particles (e.g., in quantum field theory), one builds the Fock space as a direct sum of spaces (19). For example, in a good approximation one can describe the unstable particle $\alpha$ with one decay channel $\alpha \rightarrow \beta+\gamma$, in the part of the Fock space, which includes the particle $\alpha$ itself and its decay products $\beta+\gamma$

$$
\mathscr{H}=\mathscr{H}^{(\alpha)} \oplus\left(\mathscr{H}^{(\beta)} \otimes \mathscr{H}^{(\gamma)}\right) .
$$

Each normalized state vector $|\Psi\rangle$ in the Hilbert space $\mathscr{H}$ can be represented as a sum of two orthogonal components

$$
|\Psi\rangle=|\Psi\rangle_{\alpha}+|\Psi\rangle_{\beta \gamma},
$$

where the component

$$
|\Psi\rangle_{\alpha} \equiv T|\Psi\rangle \in \mathscr{H}^{(\alpha)}
$$

lies entirely in the subspace of the unstable particle, while the other component

$$
|\Psi\rangle_{\beta \gamma} \equiv(1-T)|\Psi\rangle \in \mathscr{H}^{(\beta)} \otimes \mathscr{H}^{(\gamma)}
$$

is in the subspace of decay products. Here we denoted by $T$ the Hermitian projection on the subspace $\mathscr{H}^{(\alpha)}$

$$
T \mathscr{H}^{(\alpha)}=\mathscr{H}^{(\alpha)} .
$$

According to basic rules of quantum mechanics, the norms of these two components have simple physical interpretations:

$$
\Upsilon \equiv \||\Psi\rangle_{\alpha} \|^{2}
$$

is the probability of finding the unstable particle $\alpha$ in the state $|\Psi\rangle$, and $\||\Psi\rangle_{\beta \gamma}\left\|^{2}=1-\right\||\Psi\rangle_{\alpha} \|^{2}$ is the probability of finding its decay products. 
Observations of the unstable particle can be also described in the quantum-logical language of yes-no questions, like "Do we see the unstable particle?" and an observable, which can take two values 1 or 0 , corresponding to the possible answers "yes" or "no." Obviously, the Hermitian operator of this observable is the projection $T$ introduced above. Its eigensubspaces $\mathscr{H}^{(\alpha)}$ and $\mathscr{H}^{(\beta)} \otimes \mathscr{H}^{(\gamma)}$ correspond to the eigenvalues 1 and 0 , respectively. Then the probability of finding the particle $\alpha$ can be written as the expectation value of the projection $T$. Taking into account the property $T^{2}=T$, we see that this definition is in full agreement with (25)

$$
\begin{aligned}
\langle\Psi|T| \Psi\rangle & =(\langle\Psi| T)(T|\Psi\rangle)=\| T|\Psi\rangle\left\|^{2}=\right\||\Psi\rangle_{\alpha} \|^{2} \\
& =\Upsilon .
\end{aligned}
$$

2.5. Interacting Representation of the Poincaré Group. Next we should find out how the quantity $\Upsilon$ depends on the observer. To obtain the formula for the decay law (i.e., the time evolution of the probability $\Upsilon$ ), we can use either the Schrödinger representation, where state vectors depend on time

$$
\Upsilon(0, t)=\left(\langle\Psi| e^{(i / \hbar) H t}\right) T\left(e^{-(i / \hbar) H t}|\Psi\rangle\right),
$$

or, equivalently, the Heisenberg picture, where observables are time-dependent

$$
\Upsilon(0, t)=\left\langle\Psi\left|\left(e^{(i / \hbar) H t} T e^{-(i / \hbar) H t}\right)\right| \Psi\right\rangle .
$$

To perform these calculations, we have to specify the Hamiltonian $H$ in the Hilbert space $\mathscr{H}$. In order to keep the relativistic invariance, this Hamiltonian should be consistent with other Poincaré generators: that is, commutation relations (12)-(18) have to be satisfied.

Using available 1-particle irreducible representations $U_{g}^{(\alpha)}, U_{g}^{(\beta)}, U_{g}^{(\gamma)}$, one can easily construct one valid representation of the Poincaré group in $\mathscr{H}$

$$
U_{g}^{0} \equiv U_{g}^{(\alpha)} \oplus\left(U_{g}^{(\beta)} \otimes U_{g}^{(\gamma)}\right) .
$$

It is appropriate to call this representation "noninteracting," because its generators $\left\{\mathbf{P}_{0}, \mathbf{J}_{0}, \mathbf{K}_{0}, H_{0}\right\}$ take the forms corresponding to free particles. Apparently, in this case, the subspace $\mathscr{H}_{\alpha}$ remains invariant with respect to all inertial transformations. In particular, noninteracting translation generators commute with the projection $T$

$$
\begin{aligned}
& {\left[T, H_{0}\right]=0} \\
& {\left[T, \mathbf{P}_{0}\right]=0 .}
\end{aligned}
$$

According to Dirac and Weinberg [25, 26], one can introduce relativistic interaction by defining in $\mathscr{H}$ a new unitary representation $U_{g} \neq U_{g}^{0}$ of the Poincaré group with generators $\{\mathbf{P}, \mathbf{J}, \mathbf{K}, H\}$. Referring to our understanding of the kinematical/dynamical character of inertial transformations from Section 2.1, we can immediately conclude that generators of space translations and rotations coincide with their noninteracting counterparts

$$
\begin{aligned}
& \mathbf{P}=\mathbf{P}_{0} \\
& \mathbf{J}=\mathbf{J}_{0},
\end{aligned}
$$

while the generator of time translations contains a nontrivial interaction term $V$

$$
H=H_{0}+V
$$

(see Table 1). It is important to note that the Hermitian projection $T$ cannot commute with this interaction and with the total Hamiltonian $H$

$$
[T, H]=[T, V] \neq 0 .
$$

Indeed, only in this case, the decay law is a nontrivial function of time

$$
\begin{gathered}
\Upsilon(0,0)=\langle\Psi|T| \Psi\rangle=1 \\
e^{-(i / \hbar) H t}|\Psi\rangle \notin \mathscr{H}_{\alpha}, \quad \text { if } t \neq 0 \\
\Upsilon(0, t>0)=\left\langle\Psi\left|e^{(i / \hbar) H t} T e^{-(i / \hbar) H t}\right| \Psi\right\rangle<1
\end{gathered}
$$

as required for any unstable particle prepared at time $t=0$.

A Poincaré-Wigner-Dirac relativistic quantum description of any isolated interacting system is constructed in a similar manner. In the Hilbert space $\mathscr{H}$ of the system one defines 10 Hermitian generators $\left\{\mathbf{P}_{0}, \mathbf{J}_{0}, \mathbf{K}_{0}+\mathbf{Z}, H_{0}+V\right\}$ with commutators (12)-(18). These operators not only specify the basic total observables of the system, but also determine how the results of observations transform from one inertial system to another. Moreover, one can switch to the classical relativistic description by taking the limit $\hbar \rightarrow 0$ and considering only states describable by localized quasiclassical wave packets, which can be approximated by points in the phase space. In this limit, observables are replaced by real functions on the phase space, quantum commutators are represented by Poisson brackets, and time evolution is approximated by trajectories in the phase space $[27,28]$.

\section{Results and Discussion}

3.1. Kinematical Boosts. As we mentioned at the end of Section 2.2, Einstein's special relativity assumes that boost transformations can be represented by exact Lorentz formulas (10), which are valid universally for all events and physical systems, independent on their state, composition, and involved interactions. In other words, in special relativity boosts are kinematical.

In classical relativistic physics, this hypothesis is known as the condition of "invariant trajectories" or "manifest covariance." The well-known Currie-Jordan-Sudarshan theorem [29] states that this condition is not compatible with 
the Hamiltonian description of dynamics presented in the previous section. In other words, a Poincaré-invariant theory with invariant trajectories can exist only in the absence of interactions. This explains the name "no-interaction theorem" often used for the Currie-Jordan-Sudarshan result. Several options were tried in the literature for explaining this paradox.

One idea was that Hamiltonian dynamics is not suitable for describing relativistic interactions. Instead, various nonHamiltonian theories were developed [30-35], which deviated from the Poincaré-invariant Wigner-Dirac approach. So far, the predictive power of these theories remains rather limited.

Another idea is to abandon particles and replace them by (quantum) fields [36-39], because "there are no particles, there are only fields" [40]. This approach goes as far as claiming that there is no point in discussing observables (positions and momenta) of interacting particles, their wave functions, and also their time evolutions in the interacting regime.

The foregoing discussion suggests that the theory will not consider the time dependence of particle interaction processes. It will show that in these processes there are no characteristics precisely definable (even within the usual limitations of quantum mechanics); the description of such a process as occurring in the course of time is therefore just as unreal as the classical paths are in non-relativistic quantum mechanics. The only observable quantities are the properties (momenta, polarizations) of free particles: the initial particles which come into interaction, and the final particles which result from the process ( $L$. D. Landau and R. E. Peierls, 1930). Berestetskii et al. [41]

The more one thinks about this situation, the more one is led to the conclusion that one should not insist on a detailed description of the system in time. From the physical point of view, this is not so surprising, because in contrast to nonrelativistic quantum mechanics, the time behavior of a relativistic system with creation and annihilation of particles is unobservable. Essentially only scattering experiments are possible, therefore we retreat to scattering theory. One learns modesty in field theory. Scharf [42]

We cannot accept this point of view, because it has nothing to say about such interacting time-dependent system as the unstable particle.

3.2. Dynamical Boosts. Our preferred way to resolve the Currie-Jordan-Sudarshan controversy is to abandon the hypothesis of "invariant trajectories" and admit that boost transformations are dynamical. Actually, even in the original Dirac's paper [25], it was mentioned that, in a theory with kinematical space translations (32) and rotations (33), boosts must depend on interactions. Indeed, if we assume that boosts are kinematical $(Z=0)$, then we obtain from (17)

$$
H=\frac{i c^{2}}{\hbar}\left[K_{x}, P_{x}\right]=\frac{i c^{2}}{\hbar}\left[\left(K_{0}\right)_{x},\left(P_{0}\right)_{x}\right]=H_{0}
$$

the absurd proposition that interaction in the Hamiltonian must vanish $\left(V \equiv H-H_{0}=0\right)$.

Therefore, we should have $V \neq 0, \mathbf{Z} \neq 0$, which means that we are working in the instant form of dynamics, according to Dirac's classification [25].

3.3. Decays Caused by Boosts. Our conclusion about the dynamical character of boosts disagrees with the usual special-relativistic "geometrical" view on boosts. In particular, we can no longer claim that

Any event that is "seen" in one inertial system is "seen" in all others. For example if observer in one system "sees" an explosion on a rocket then so do all other observers. Polishchuk [21]

Returning to our example of unstable particle, we can say that when the observer at rest sees the pure unstable particle $\alpha$, moving observers may see also its decay products $\beta+\gamma$ with some probability. We can prove an even stronger statement: if all (both resting and moving with different rapidities $\boldsymbol{\theta}$ ) observers see the particle $\alpha$ at $t=0$ with $100 \%$ probability

$$
\Upsilon(\theta, 0)=1
$$

then this particle is stable with respect to time translations as well.

Suppose that (38) is true; that is, for any $|\Psi\rangle \in \mathscr{H}_{\alpha}$,

$$
\Upsilon(\theta, 0) \equiv\left\langle\Psi\left|e^{-(i c / \hbar) K_{x} \theta} T e^{(i c / \hbar) K_{x} \theta}\right| \Psi\right\rangle=1 .
$$

This means that all boosts leave the subspace $\mathscr{H}_{\alpha}$ invariant

$$
e^{(i c / \hbar) K_{x} \theta}|\Psi\rangle \in \mathscr{H}_{\alpha}, \quad \forall \theta
$$

and that the interacting boost operator $K_{x}$ commutes with the projection $T$. Then commutators (17) and (31) and the Jacobi identity imply

$$
\begin{aligned}
{[T, H] } & =-\frac{i c^{2}}{\hbar}\left[T,\left[K_{x}, P_{0 x}\right]\right] \\
& =\frac{i c^{2}}{\hbar}\left[K_{x},\left[P_{0 x}, T\right]\right]+\frac{i c^{2}}{\hbar}\left[P_{0 x},\left[T, K_{x}\right]\right]=0
\end{aligned}
$$

which contradicts the fundamental property (35) of unstable particles. To resolve this contradiction, we have to admit that the boosted state $e^{(i c / \hbar) K_{x} \theta}|\Psi\rangle$ does not correspond to the particle $\alpha$ with $100 \%$ probability. This state must contain an admixture of decay products even at the initial time $t=0$

$$
e^{(i c / \hbar) K_{x} \theta}|\Psi\rangle \notin \mathscr{H}_{\alpha}, \quad \text { if } \theta \neq 0 .
$$


This means that, from the point of view of the moving observer, the state vector's projection on the subspace of decay products is nonzero

$$
\begin{aligned}
0 & <\|(1-T) e^{(i c / \hbar) K_{x} \theta}|\Psi\rangle \|^{2} \\
& =\left\langle\Psi\left|e^{-(i c / \hbar) K_{x} \theta}(1-T)(1-T) e^{(i c / \hbar) K_{x} \theta}\right| \Psi\right\rangle \\
& =\left\langle\Psi\left|e^{-(i c / \hbar) K_{x} \theta}(1-T) e^{(i c / \hbar) K_{x} \theta}\right| \Psi\right\rangle \\
& =1-\left\langle\Psi\left|e^{-(i c / \hbar) K_{x} \theta} T e^{(i c / \hbar) K_{x} \theta}\right| \Psi\right\rangle
\end{aligned}
$$

and that the nondecay probability is less than unity

$$
\Upsilon(\theta, 0)=\left\langle\Psi\left|e^{-(i c / \hbar) K_{x} \theta} T e^{(i c / \hbar) K_{x} \theta}\right| \Psi\right\rangle<1 .
$$

This is the "decay caused by boost" $[11,12,43]$, which means, among other things, that special-relativistic formulas (1) and (38) are inaccurate, and that boosts have a nontrivial effect on the internal state of the unstable particle.

It is important that (44) describes decays viewed from a moving reference frame, that is, by a moving detector. This is completely different from the more familiar experimental setup in which a stationary detector looks at a moving particle. In the latter case, the state vector of the moving unstable particle lies entirely in the subspace $\mathscr{H}_{\alpha}$, and there is no "decay caused by boost."

3.4. Discussion. Here we discussed the dynamical effect of boosts on unstable particles (44). However, similar nontraditional effects should be visible also in other interacting systems, even in classical (nonquantum) ones [44-46]. In order to verify these predictions, one has to look at composite interacting systems, where interaction acts for a sufficiently long time. Unfortunately, most experimental checks of special relativity [47-49] do not satisfy these criteria. For example, dynamical boosts do not change the relativistic kinematics (the relationships between momenta, velocities, and energies of free particles) in collisions, reactions, and decays. Likewise, dynamical boosts do not affect Doppler type experiments $[50,51]$, which measure the frequency (energy) of light and its dependence on the motion of the source or the observer. Michelson-Morley type experiments [52-54], studying the invariance of the speed of light, are not affected as well.

The time dilation experiments with unstable particles [1-4] are exceptional, because they study systems that are under the action of interaction during sufficiently long time interval. Unfortunately, predicted deviations from the special-relativistic time dilation formula (1) are too small to be observed. One can see that the "decay caused by boost" effect is also very small and beyond the sensitivity of modern experiments [12].

Perhaps, the most convincing evidence for the dynamical character of boosts was obtained in the Frascati experiment [55-57], which established the superluminal dynamics of the electric field of relativistic charges. This observation was explained from the point of view of the Poincaré-WignerDirac theory in $[27,58,59]$.

\section{Conclusions}

We applied Poincaré-Wigner-Dirac theory of relativistic interactions to unstable particles. In particular, we were interested in how the same particle is seen by different moving observers. We proved that the decay probability cannot be invariant with respect to boosts. Different moving observers may see different internal compositions of the same particle. In spite of being very small, this effect is fundamentally important as it sets the limit of applicability for special relativity.

\section{Conflicts of Interest}

The author declares no conflicts of interest.

\section{References}

[1] B. Rossi and D. B. Hall, "Variation of the rate of decay of mesotrons with momentum," Physical Review A: Atomic, Molecular and Optical Physics, vol. 59, no. 3, pp. 223-228, 1941.

[2] D. H. Frisch and J. H. Smith, "Measurement of the relativistic time dilation using $\mu$-mesons," American Journal of Physics, vol. 31, no. 5, article 342, 1963.

[3] D. S. Ayres, A. M. Cormack, A. J. Greenberg et al., "Measurements of the lifetimes of positive and negative pions," Physical Review D: Particles, Fields, Gravitation, and Cosmology, vol. 3, article 1051, 1971.

[4] C. E. Roos, J. Marraffino, S. Reucroft et al., " $\sigma^{ \pm}$lifetimes and longitudinal acceleration,” Nature, vol. 286, pp. 244-245, 1980.

[5] J. Bailey, K. Borer, F. Combley et al., "Measurements of relativistic time dilatation for positive and negative muons in a circular orbit," Nature, vol. 268, pp. 301-305, 1977.

[6] F. J. M. Farley, "The CERN (g-2) measurements," Zeitschrift für Physik C Particles and Fields, vol. 56, supplement 1, pp. S88-S96, 1992.

[7] L. A. Khalfin, Quantum Theory of Unstable Particles and Relativity, 1997, preprint of Steklov Mathematical Institute, St. Petersburg Department, PDMI-6/1997, http://www.pdmi.ras.ru/ preprint/1997/97-06.html.

[8] K. Urbanowski, "The true face of quantum decay processes: unstable systems at rest and in motion," Acta Physica Polonica $B$, vol. 48, no. 10, article 1411, 2017.

[9] G. N. Fleming, "Observations on unstable quantons, hyperplane dependence and quantum fields," Studies in History and Philosophy of Science. Part B. Studies in History and Philosophy of Modern Physics, vol. 42, no. 2, pp. 136-147, 2011.

[10] M. Shirokov, "Decay law of moving unstable particle," International Journal of Theoretical Physics, vol. 43, no. 6, pp. 1541-1553, 2004.

[11] E. V. Stefanovich, "Quantum effects in relativistic decays," International Journal of Theoretical Physics, vol. 35, no. 12, pp. 2539-2554, 1996.

[12] E. V. Stefanovich, "Violations of Einstein's time dilation formula in particle decays," 2006, https://arxiv.org/abs/physics/0603043.

[13] P. Exner, "Representations of the Poincaré group associated with unstable particles," Physical Review D: Particles, Fields, Gravitation and Cosmology, vol. 28, no. 10, pp. 2621-2627, 1983.

[14] S. A. Alavi and C. Giunti, "Which is the quantum decay law of relativistic particles?" Europhysics Letters, vol. 109, no. 6, article 60001, 2015. 
[15] E. V. Stefanovich, "Is Minkowski space-time compatible with quantum mechanics?" Foundations of Physics. An International Journal Devoted to the Conceptual Bases and Fundamental Theories of Modern Physics, vol. 32, no. 5, pp. 673-703, 2002.

[16] A. R. Lee and T. M. Kalotas, "Lorentz transformations from the first postulate," American Journal of Physics, vol. 43, no. 5, article 434, 1975.

[17] J.-M. Lévy-Leblond, "One more derivation of the Lorentz transformation," American Journal of Physics, vol. 44, no. 3, pp. 271-277, 1976.

[18] D. A. Sardelis, "Unified derivation of the Galileo and the Lorentz transformations," European Journal of Physics, vol. 3, no. 2, article 96, 1982.

[19] H. M. Schwartz, "Deduction of the general Lorentz transformations from a set of necessary assumptions," American Journal of Physics, vol. 52, no. 4, article 346, 1984.

[20] J. H. Field, "A new kinematical derivation of the Lorentz transformation and the particle description of light," Helvetica Physica Acta, vol. 70, pp. 542-564, 1997, https://arxiv.org/abs/ physics/0410262.

[21] R. Polishchuk, "Derivation of the Lorentz transformations," 2001, https://arxiv.org/abs/physics/0110076.

[22] A. Galiautdinov, "Derivation of the Lorentz transformation without the use of Einstein's second postulate," 2017, https://arxiv.org/abs/1701.00270.

[23] E. P. Wigner, Group Theory and its Application to the Quantum Mechanics of Atomic Spectra, Academic Press, New York, NY, USA, 1959.

[24] E. Wigner, "On unitary representations of the inhomogeneous Lorentz group," Annals of Mathematics, vol. 40, no. 1, pp. 149204, 1939 .

[25] P. A. M. Dirac, "Forms of relativistic dynamics," Reviews of Modern Physics, vol. 21, article 392, 1949.

[26] S. Weinberg, The Quantum Theory of Fields, vol. 1, Cambridge University Press, Cambridge, UK, 1995.

[27] E. V. Stefanovich, "Relativistic quantum dynamics: a non-traditional perspective on space, time, particles, fields, and actionat-a-distance," 2005, https://arxiv.org/abs/physics/0504062.

[28] E. Stefanovich, Relativistic Quantum Theory of Particles, vol. I, Lambert Academic Publishing, Saarbrücken, Germany, 2015.

[29] D. G. Currie, T. F. Jordan, and E. C. Sudarshan, "Relativistic invariance and Hamiltonian theories of interacting particles," Reviews of Modern Physics, vol. 35, pp. 350-375, 1963.

[30] H. Van Dam and E. P. Wigner, "Classical relativistic mechanics of interacting point particles," Physical Review A: Atomic, Molecular and Optical Physics, vol. 138, no. 6B, pp. B1576-B1582, 1965.

[31] H. Van Dam and E. P. Wigner, "Instantaneous and asymptotic conservation laws for classical relativistic mechanics of interacting point particles," Physical Review A: Atomic, Molecular and Optical Physics, vol. 142, no. 4, pp. 838-843, 1966.

[32] E. C. G. Sudarshan and N. Mukunda, "Forms of relativistic dynamics with world line condition and separability," Foundations of Physics, vol. 13, no. 3, pp. 385-393, 1983.

[33] W. N. Polyzou, "Manifestly covariant, Poincaré-invariant quantum theories of directly interacting particles," Physical Review D: Particles, Fields, Gravitation and Cosmology, vol. 32, no. 4, pp. 995-1003, 1985.

[34] B. D. Keister, "Forms of relativistic dynamics: what are the possibilities?" AIP Conference Proceedings, vol. 334, no. 1, article $164,1995$.
[35] S. N. Sokolov, "Mechanics with Retarded Interactions," 1997, preprint IHEP Protvino, IHEP 97-84, http://web.ihep.su/library/ pubs/prep1997/ps/97-84.pdf.

[36] D. B. Malament, "In defense of dogma: why there cannot be a relativistic quantum mechanics of (localizable) particles," in Perspectives on Quantum Reality, R. Clifton, Ed., Springer, Dordrecht, Netherlands, 1996.

[37] F. Wilczek, "Quantum field theory," Reviews of Modern Physics, vol. 71, no. 2, pp. S85-S95, 1999.

[38] H. Halvorson and R. Clifton, "No place for particles in relativistic quantum theories?" in Ontological Aspects of Quantum Field Theory, pp. 181-213, 2002.

[39] F. Strocchi, "Relativistic quantum mechanics and field theory," Foundations of Physics, vol. 34, no. 3, pp. 501-527, 2004.

[40] A. Hobson, "There are no particles, there are only fields," American Journal of Physics, vol. 81, no. 3, article 211, 2013.

[41] V. B. Berestetskii, E. M. Livshitz, and L. P. Pitaevskii, Quantum Electrodynamics, Elsevier, Oxford, UK, 1982.

[42] G. Scharf, Finite Quantum Electrodynamics. The Causal Approach, Texts and Monographs in Physics, Springer-Verlag, Berlin, Germany, 2nd edition, 1995.

[43] F. Giacosa, "Decay law and time dilatation," Acta Physica Polonica B, vol. 47, no. 9, pp. 2135-2150, 2016.

[44] W. Glöckle and Y. Nogami, "Relativistic dynamics and Lorentz contraction," Physical Review D: Particles, Fields, Gravitation and Cosmology, vol. 35, no. 12, pp. 3840-3846, 1987.

[45] B. Hamme and W. Glöckle, "Relativistic two-body bound state in motion," Few-Body Systems, vol. 13, no. 1, pp. 1-10, 1992.

[46] B. T. Shields, M. C. Morris, M. R. Ware, Q. Su, E. V. Stefanovich, and R. Grobe, "Time dilation in relativistic two-particle interactions," Physical Review A: Atomic, Molecular and Optical Physics, vol. 82, no. 5, Article ID 052116, 2010.

[47] D. Newman, G. W. Ford, A. Rich, and E. Sweetman, "Precision experimental verification of special relativity," Physical Review Letters, vol. 40, no. 21, pp. 1355-1358, 1978.

[48] D. W. MacArthur, "Special relativity: understanding experimental tests and formulations," Physical Review A: Atomic, Molecular, and Optical Physics and Quantum Information, vol. 33, no. 1, 1986.

[49] C. M. Will, "Special relativity: a centenary perspective", in Einstein, 1905-2005, T. Damour, O. Darrigol, B. Duplantier, and V. Rivasseau, Eds., vol. 47 of Progress in Mathematical Physics, Birkhäuser, Basel, Switzerland, 2005.

[50] M. Kaivola, O. Poulsen, E. Riis, and S. A. Lee, "Measurement of the relativistic doppler shift in Neon," Physical Review Letters, vol. 54, no. 4, pp. 255-258, 1985.

[51] D. Hasselkamp, E. Mondry, and A. Scharmann, "Direct observation of the transversal Doppler-shift," Zeitschrift füur Physik A Atoms and Nuclei, vol. 289, no. 2, pp. 151-155, 1979.

[52] H. Müller, C. Braxmaier, S. Herrmann, A. Peters, and C. Lämmerzahl, "Electromagnetic cavities and Lorentz invariance violation," Physical Review D: Particles, Fields, Gravitation and Cosmology, vol. 67, no. 5, Article ID 056006, 2003.

[53] P. Wolf, S. Bize, A. Clairon, A. N. Luiten, G. Santarelli, and M. E. Tobar, "Tests of Lorentz invariance using a microwave resonator," Physical Review Letters, vol. 90, no. 6, Article ID 060402, 2003.

[54] T. Alväger, F. J. M. Farley, J. Kjellman, and L. Wallin, “Test of the second postulate of special relativity in the $\mathrm{GeV}$ region," Physics Letters, vol. 12, no. 3, pp. 260-262, 1964. 
[55] R. de Sangro, G. Finocchiaro, P. Patteri, M. Piccolo, and G. Pizzella, "Measuring propagation speed of Coulomb fields," The European Physical Journal C, vol. 75, no. 3, 2015.

[56] R. de Sangro, G. Finocchiaro, P. Patteri, M. Piccolo, and G. Pizzella, "Why the interpretation of "Measuring propagation speed of Coulomb fields" stands," The European Physical Journal C, vol. 77, no. 2, 2017.

[57] R. de Sangro, G. Finocchiaro, P. Patteri, M. Piccolo, and G. Pizzella, "Experimental result on the propagation of Coulomb fields," Journal of Physics: Conference Series, vol. 845, Article ID 012015, 2017.

[58] E. Stefanovich, Relativistic Quantum Theory of Particles, vol. II, Lambert Academic Publishing, Saarbrücken, Germany, 2015.

[59] E. V. Stefanovich, "Does Pizzella’s experiment violate causality?" Journal of Physics: Conference Series, vol. 845, Article ID 012016, 2017. 

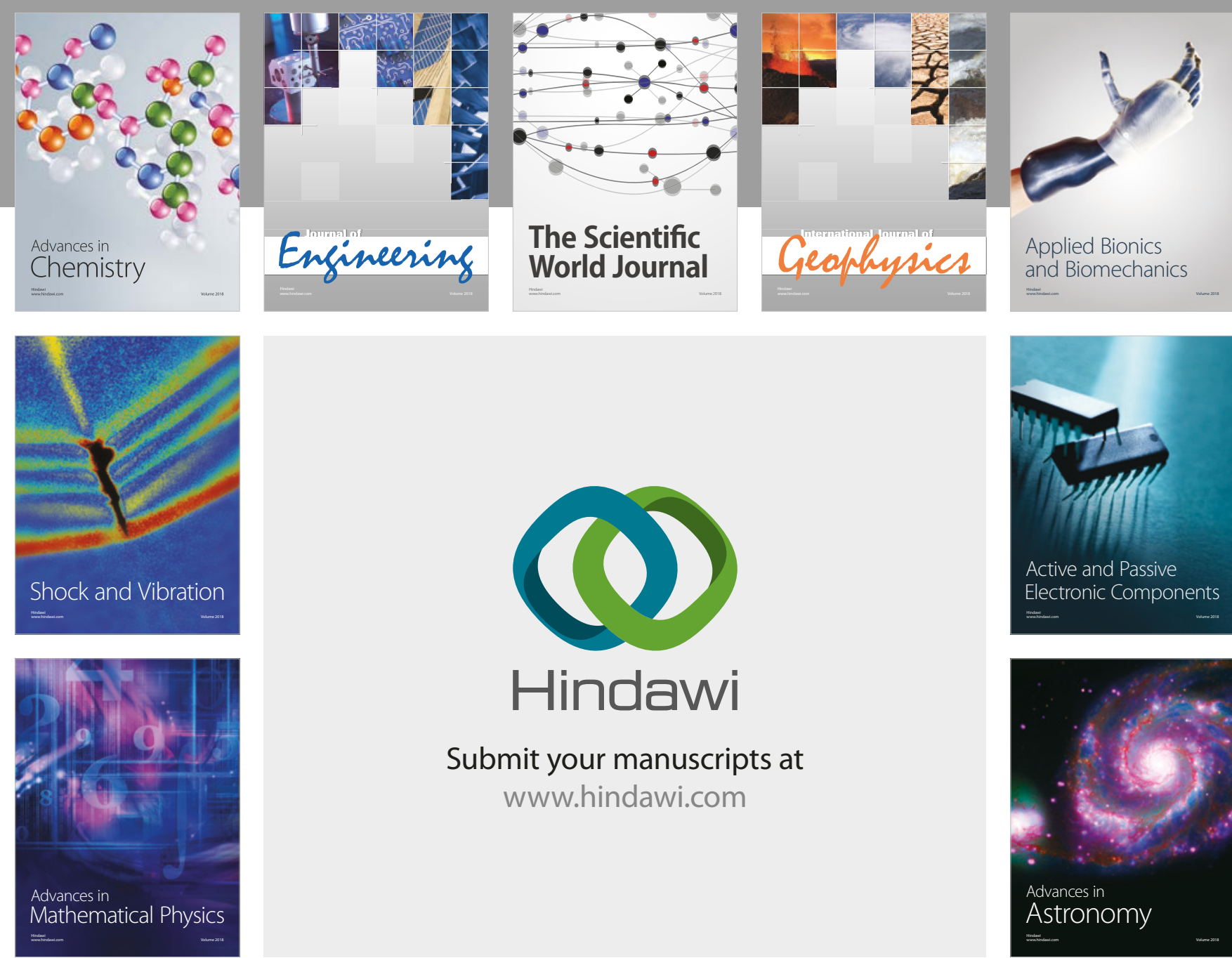

Submit your manuscripts at

www.hindawi.com

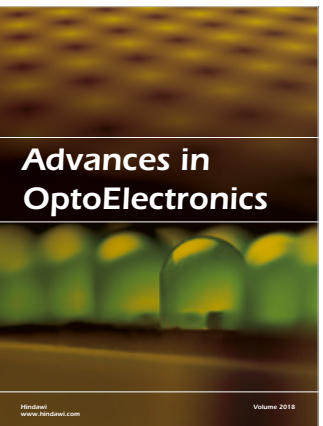

\section{Rotcting Machinery}
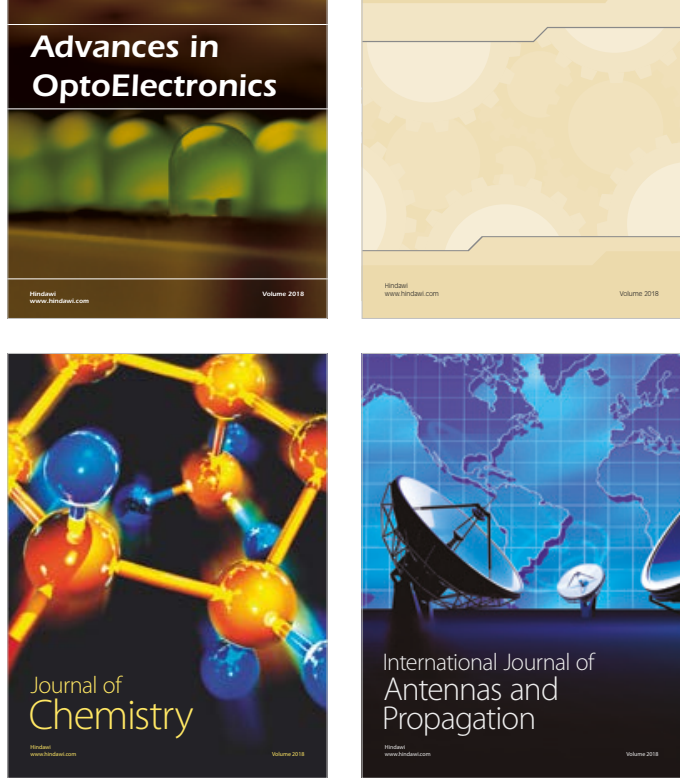

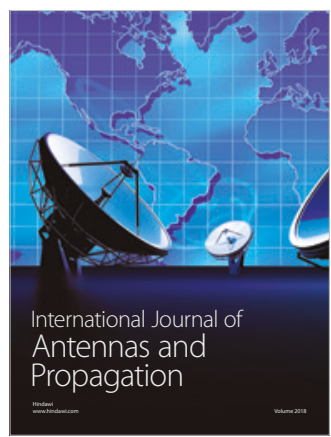

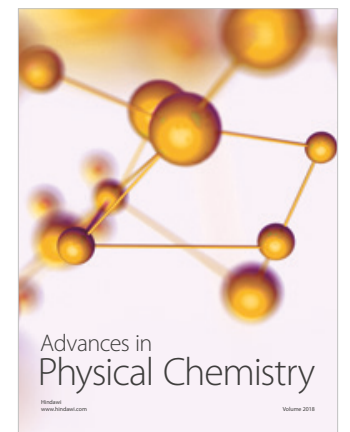

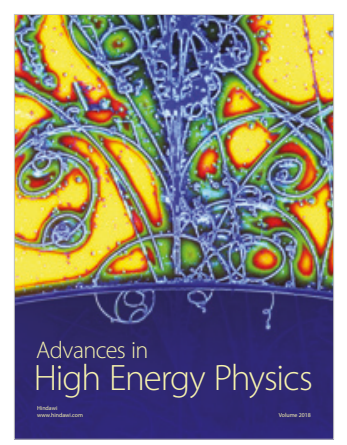

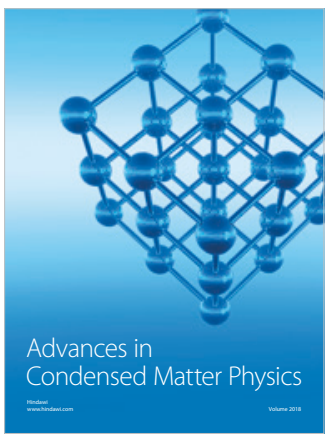

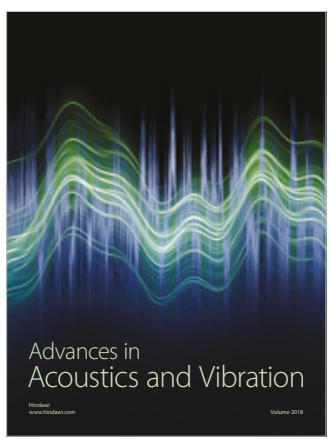

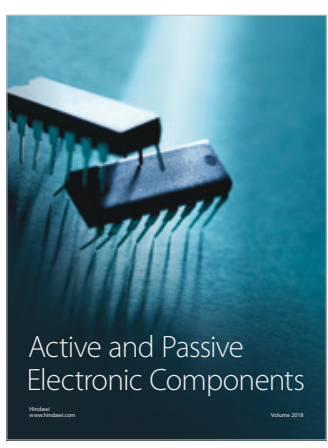
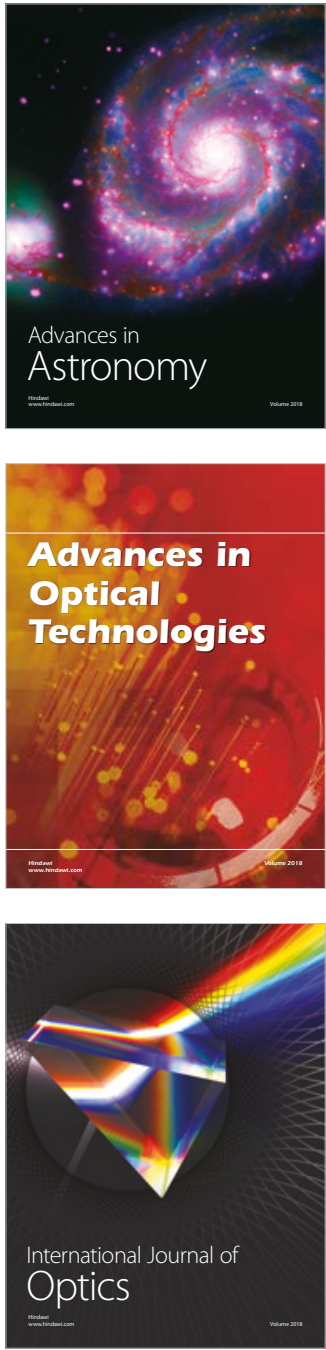